Aus der I. Medizinischen Abteilung der k. k. Krankenanstalt „Rudolfstiftung" in Wien.

\section{Zur radiologischen Diagnose des Magen- und Duodenalgeschwürs.}

Die gestrigerte Aufmerksamkeit, welche dem Vorkommen von Magen-, besonders aber Zwסlffingerdarmgeschwüren in den letzten Jahren von seiten der Klinik zugewendet wird, hat mit dem Ausbau der Diagnostik dieser Erkrankungen auch eine Revision der Röntgendiagnose zur Folge gehabt.

Schon die bekanntesten Autoren in der Radiologie des Magengeschwürs: Faulhaber, Holzknecht, Rieder, Schmieden, Jonas, Haudek, Schwarz u. A. haben bei der Suche nach entscheidenden Kriterien für den Nachweis der verschiedenen Geschwürsformen immer wieder auf die differentialdiagnostische Seite gegenüber anderen Magenerkrankungen mit ähnlichen $\mathrm{Be}$ funden hingewiesen. Bei dem Ueberblick über die zahlreichen Arbeiten, besonders aber in der wertvollen monographischen Darstellung über die klinische Röntgendiagnostik des Verdauungskanals von Stierlin, kann man das Bestreben konstatieren, nach zahlreichen sichergestellten Betunden und Beobachtungen die diagnostische Beweiskraft der am Röntgenschirm wahrnehmbaren Veränderungen zu bewerten.

So eindeutig sich die Befunde beim kallösen penetrierenden Magengeschwür, die Befunde der Magennische von $\mathrm{Haudek}$ darstellen, so läßt zweifellos die radiologische Erkenntnis des einfachen Magengeschwürs noch viele Wünsche übrig. Für jeden Kenner dieser Verhältnisse genügt diese Konstatierung, und es ist unnötig, im einzelnen auf die immer wiederkehrenden Lücken in der Erkenntnis und die Fehloperationen hinzuweisen.

Noch viel auffälliger, weil erst in der jüngsten Zeit in Entwicklung begriffen, sind die Ergebnisse der Radiologie des Duodenalgeschwürs. Ich möchte in dieser so aktuellen Frage hier nicht ins Detail gehen und behalte mir dies für eine zusammenhängende Darstellung an anderem Orte vor.

Seit Moynihans imponierenden Befunden beginnt in unseren Landen ein Wettlauf nach dem Duodenalgeschwür, welches fast zur Modekrankheit der Aerzte sich zu entwickeln droht. Damit soll natürlich die große Häufigkeit des früher übersehenen Duodenalgeschwürs nicht in Abrede gestellt, aber auf die Mängel der Untersuchungsmethoden und eine gewisse Leichtfertigkeit bei der Annahme dieser Erkrankung hingewiesen werden. Das viel gegeißelte Wort Moynihans „Die Anamnese ist Alles, die objektive Untersuchung nichts" ist oft genug an den Pranger gestelli worden. Die Gegenreaktion, das Suchen nach objektiven Symptomen, ist nicht ausgeblieben und hat eine Menge von Versuchen gebracht, das Duodenalgeschwür mit

*) Das ist nicht etwa eine bloße Vermutung von mir, sondern durch den Versuch am Menschen erwissen. Rhe a Barto $\mathbf{n}$ (On the treatment of anchylosis by the formation of artificial joints, Philadelphia 1827) fuihrte seine klassische Operation, die Osteotomia subtrochanterica, nicht etwa aus der jetzt allgemein gültigen Indikation aus, um verkehrte Stellungen des Beines nach Hüftankylose zu verbessern, sondern er wollte durch methodische Bewegungen eine Pseudarthrose erzeugen, wie schon der Titel seiner Veröffentlichung besagt. Das ist niemals gelungen, wohl aber hat $\mathrm{R} h$ e a $\mathrm{B}$ art on trotz dieses Mißerfolges seiner Absicht eine außerordentlich wichtige Operation erfunden.

eindeutigen radiologischen Erkennungszeichen auszustatten. Gerade die Erscheinungen des Krieges, der eine Häufung von wirklichen und ulkusverdächtigen Kranken gebracht hat, haben unsere Erfahrungen auf dem Gebiete des Erkennens und Verkennens des Duodenalgeschwürs wesentlich berfichert. Ich möchte in diesem kleinen Beitrag, der nichts weniger als vollständig sein soll, nur einige Hinweise auf die Fehlerquelle n bei der Diagnose dieser Geschwürsformen bringen. Ich will dabei das Geständnis nicht unterlassen, daß ich aus eigenen Fehlern gelernt habe. Es wäre für die Klärung dieser Fragen von größtem Vorteil, wenn man es sich allgemein zur Gewohnheit machen würde, Versehen einzugestehen und aus ihrer Berichtigung die heilsame Kritik abzuleiten. Nichts wirkt erziehlicher als diese Art der Betrachtung, und darum allein schon halte ich den Weg der Belehrung und Aufklärung, wie' ihn Schwalbe in seinem neuen Sammelwerke „Diagnostische und therapeutische Irrtümer" eingeschlagen hat, für verheißungsvoll und aussichtsreich.

Ein Wort nur von der Bedeutung der A namnese. In der Hand des geübten und nüchternen Beurteilers kann sie uns ja sehr viel sagen. Weit gefährlicher erweist sie sich in der Hand des Anfängers. Die Suche nach dem Hungerschmerz, dem Spätschmerz, dem Nüchternschmerz usw. ist zu einer förmlichen monomanischen Einstellung der Aerzte geworden. Was man alles an anamnestischen Angaben in einen Kranken hineinsuggerieren kann, ist ja bekannt. Und mit wachsendem Erstaunen hören wir jetzt Tag für Tag, wie uns unaufgefordert der. einfache Mann aus dem Volke die Beobachtungen Moynihans als seine Klagen vorbringt. Es ist kein Zweifel, auch die Kranken sind schon von der neuen Lehre befruchtet; sonst wäre es nicht möglich, daß wir früher an diesen vielen eindringlichen anamnestischen Mitteilungen achtlos vorbeigegangen wären. Schon Kehr hat darauf hingewiesen, daß ein echter Hungerschmerz bei der Cholelithiasis vorkommt. Ich habe am Londoner Kongreß die Aehnlichkeit des klinischen Verhaltens beider Affektionen betont, und es sei nur des Interesses halber nochmals angeführt, daß $\mathrm{Kehr}$ die rhythmische Entleerung der gefüllten Gallenblase, welche reflektorisch von der Magenfüllung aus angeregt wird, mit der Genese des Hungerschmerzes in diesen Fällen in Beziehung bringt. Bei großen Intervallen zwischen den einzelnen Mahlzeiten erreicht die Füllung der Gallenblase eine größere Intensität. Das chronisch entziundete oder vielfach adhärente Organ wird in seiner Wand gezerrt und gespannt, und es entstehen Schmerzen, die sich mit dem Andauern des Hungers steigern. Die Magenfüllung ruft reflektorisch die Entleerung der Gallenblase hervor und beseitigt die Ursache des Schmerzes. Von den vielen Verwechslungsmöglichkeiten des Hungerschmerzes und der Mo y ni ha n schen Anamnese möchte ich einen recht lehrreichen Fall anführen, der durch die Operation bestätigt wurde.

Herr A. H., Ingenieur, 45 Jahre alt, ist mir seit Jahren bekannt. Er litt früher wiederholt an Darmkatarrhen. Im Dezember 1915 erkrankte er an sehr heftigen Beschwerden, wehenartigen Schmerzen im rechten Hypochondrium, 4-4 $\mathbf{4}^{1 / 2}$ Stunden nach der Mahlzeit, welche durch Nahrungsaufnahme, namentlich durch kompakte Nahrung, gemildert werden. Sodbrennen. Wiederholt haben die Attacken solche Intensität erreicht, daß ein herbeigerufener Arzt Morphininjektionen geben mußte. Mehrmals Erbrechen im Anfall. Einmal Ohnmachtsanfall "im Schmerz. Stuhl wiederholt dunkel. Die Schmerzen treten in der letzten' Zeit jede Nacht auf. Untersuchung auf okkulte Blutung negativ. Ausheberung des Magens konnte wegen hochgradiger Reizbarkeit des Kranken nicht ausgeführt werden. Röntgenuntersuchung (Prof. Holzknecht) ergibt einen hypertonischen und hypermobilen Magen; an diesem und am Duodenum keine zirkumskripten Veränderungen sichtbar, keine Druckempfindlichkeit. Das Zökum dystopisch, adhepatal gelegen, sodaß eng gedrängt an einer Stelle des rechten oberen Bauchquadranten dàs Zökum, das Duodenum und die Gallenblase kollidieren ohne Differenzierbarkeit. Oberes Kolon hypermobil. Die Umgebung des. Zökums, Pylorus und Pars superior duodeni erweist sich vor dem Schirm als druckempfindlich (siehe Fig. 1)

Obwohl die Symptome eine Duodenal- resp. Gallenblasenaffektion nahelegten, entschied ich mich doch, nach den Antezedentien, für die Diagnose einer chronischen Ap'pendizitis bei dystopischem Zökum. Die von Prof. A. Lorenz am 17. II. 16 vorgenommene Operation ergab eine vollkommene Bestätigung der radiologisch er- 
Fig. 1.

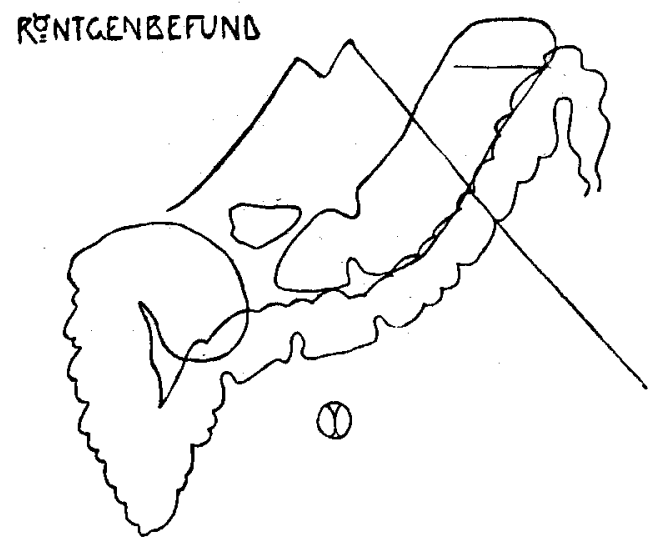

hobenen Lageanomalie des Zökums, eine innige Verwachsung der Pars superior duodeni mit der Gallenblase; Duodenum, Pylorus und Gallenblase frei von Veränderungen. Das auf den Kopf gestellte, mit der Unterfläche der Leber verwachsene Zökum wird abpräpariert und der mit der Umgebung verbackene, in seiner Wand verdickte, im Lumen verringerte Wurmfortsatz abgetragen. Das mobilisierte Zökum wird heruntergezogen und in der rechten Darmbeingrube an die vordere Bauchwand fixiert. (Zökopexie.) Heilung.

Fig. 8.

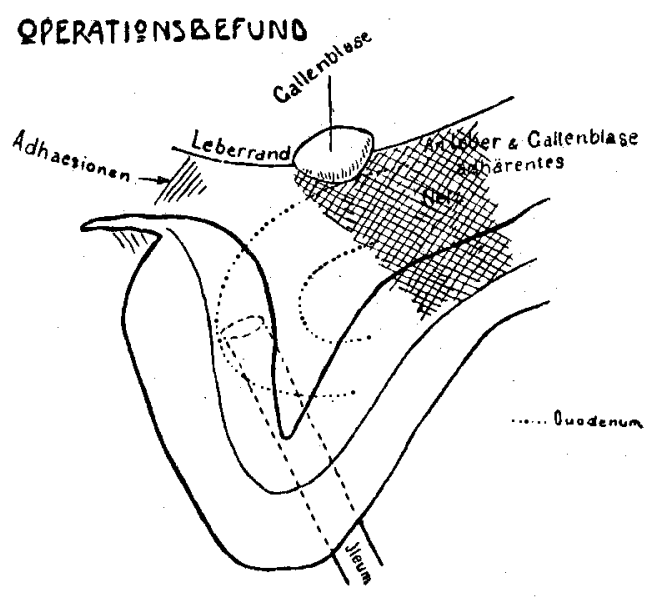

Die typischen Beschwerden in diesem Falle wiesen zwar aut die Duodenalgegend hin, sind jedoch durch eine ganz anders geartete Affektion hervorgerufen worden. Die Erkenntnis der Sachlage vor der Operation in diesem Falle ist zweifellos ein Verdienst der Röntgenuntersuchung.

Wie weit die Uebereinstimmung im Symptomenbilde des Duodenalgeschwürs und der Grllenblasenerkrankung geht, ist genügend bekannt. Die atypische Cholelithiasis, die Schmerzen bei der chronischen Gallensteinkrankheit, die Reflexsymptome am Magen, auf deren radiologische Erkennung zuerst Emmo Schlesinger aufmerksam gemacht hat, das häufige Vorkommen von Hunger- und Nüchternschmerz, Nachtschmerz bei den Erkrankungen der Gallenblase, erklären nur zu leicht die Verwechslung dieser Erkrankungen mit den Duodenalaffektionen. Schon vor vier Jahren hat Friedrich Kraus darauf aufmerksam gemacht, daß auch bei genauer radiologischer Untersuchung das Auseinanderhalten der Gallenblasen- und Duodenalaffektionen mitunter unmöglich ist und Fehldiagnosen nach beiden Seiten hin oft unvermeidlich sind. Die Interferenz beider Affektionen wird dadurch noch inniger, daß sekundäre Verwachsungen, Verlötungen des Duodenums mit der Gallenblase als Entzündungsfolge bei Cholezystitis so häufig beobachtet werden. Dazu kommt eine mir längst bekannte klinische Erfahrung, auf welche Na u y n schon hingewiesen hat, daß auch bei der komplizierten chronischen Gallensteinkrankheit zeitweise bluthaltige Stühle auftreten.

Bei der Durchmusterung meines großen Materials von Kriegskranken ist mir aufgefallen, wie oft ich und andere zu Beginn des Krieges an den zahlreichen verdauungskranken Soldaten Geschwürssymptome, und besonders die Erscheinungen des
Duodenalgeschwürs, festgestellt haben. Das Zusammentreffen klinischer und radiologischer Merkmale von Duodenalgeschwür hat sich bei einer großen Zahl von Soldaten so auffallend wiederholt, daß man stutzig werden mußte. Eine ganze Anzahl dieser Fälle konnte durch weitere Beobachtung aufgeklärt werden; wiederholt brachten Rezidive die entlassenen, ins Feld abgegangenen Kranken wieder in unsere Beobachtung zurück, und die Veränderung des Krankheitsbildes hat uns eine Richtig. stellung ermöglicht. Ein Ma nifestwerden latenter Gallenbeschwerden durch Schwellung der Leber; Auftreten einer tastbaren, schmerzhaften Gallenblase, Urobilinikterus resp. Auftreten von Urobilinurie und Urobilinogenurie führten zur einwandfreien Aufklärung. Die genaue Durchsicht ergab, daß es sich fast durchwegs um Kriegsteilnehmer handelte, die eine der überaus häufigen infektiösen Darmerkrankungen aus der KoliTyphusgruppedurchgemacht hatten. Dysenterie oder dysenterieähnliche Erkrankungen, namentlich aber der Paratyphus - und selbst kurzdauernde Diarrhöen mit bluthaltigen Stühlen sind für eine solche Infektion in hohem Grade verdächtig führen in der Regel zu ulzerösen Veränderungen im Dick- und Dünndarm. Wenn auch die Geschwürsbildung abheilt, bleibt, wie die endoskopische Besichtigung des Darmes lehrt, in der überaus großen Mehrzahl solcher Fälle eine leichte Vulnerabilität der Darmschleimhaut zurück, und kleinste kapilläre Blutungen sind ein häufiger Befund. Kein Wunder, daß hier so oft der Nachweis auf okkultes Blut im Stuhl positiv ausfällt, der im Verein mit den übrigen charakteristischen Magenbeschwerden auf ein Magen- resp. Duodenalgeschwür bezogen wird. Die Röntgenveränderungen: Hypertonie und Hyperperistaltik des Magens, Sechsstundenrest, durch Pylorospasmus hervorgerufen, ja so häufig Einkerbungen am Bulbus duodeni, denen keine anatomischen Veränderungen entsprechen (spastische Einziehungen), sind der Ausdruck der motorischen Mitbeteiligung des Magen-Duodenaltraktes bei der chronischen Gallenblasenaffektion. Diese Gallenblasenerkrankung ist, wie bekannt, eine Lieblingslokalisation der Bakterien aus der Typhus-Koli-Gruppe, und bei jugendlichen, männlichen Individuen ist diese Infektion eine der häufigsten Ursachen der chronischen Gallenblasenentzündung.

Fast ebenso häufig bringt die latente oder chronische Lungent uberkulose mit Hervortreten gastrischer Beschwerden einen klinischen Komplex hervor, der zur Verwechslung mit Magen- resp. Duodenalgeschwüren führt. Ich habe auf dieses eigentümliche Zusammentreffen vor zwei Jahren aufmerksam gemacht, ${ }^{1}$ ) indem ich aus einer Gruppe eigener Beobachtungen einen Fall von Pylorospasmus und Magenblutung mitteilte, bei dem ich durch lang fortgesetzte. Beobachtung den wechselartigen Verlauf der Erkrankung bis zum Tode verfolgen konnte.

Ein junges, mit Tuberkulose hereditär belastetes Mädchen, das seit jeher an „,schwachem Magen" litt, kam mit Erbrechen, qualvollen Schmerzen im Epigastrium und Erscheinungen hochgradiger Erregbarkeit zur Zeit der Menstruation in meine Beobachtung. Die Details der Krankengeschichte sind in der ersten Originalmitteilung nachzulesen. Damals wurden die Erscheinungen des erhöhten Vagustonus klinisch und durch den Pilokarpinversuch und eine Magenneurose durch Erkrankung des vegetativen Systems festgestellt. Nach Entlassung aus meiner Beobachtung Verschlechterung des Zur standes, wiederholtes Bluterbrechen, das sie zu einem Chirurgen führt, der einen operativen Eingriff unter der Annahme des Magengeschwürs vornahm, bestärkt durch einen typischen, für Olcus pylori sprechenden Röntgenbefund. Bei der Operation fand sich Fehlen aller Anzeichen von Ulkus, Hypertonie des Magens und Spasmen am Pylorus. Bald nach der Operation Wiederkehr aller Beschwerden, Schmerzen, Erbrechen, Bluterbrechen, soda $\beta$ die Patientin neuerdings an meiner Abteilung Hilfe sucht. Elender Ernährungszustand, Erbrechen, Schmerzen, schlechte Funktion der Gastroanastomose charakterisierten den Zustand der Kranken, bei welcher nach vorübergehender Besserung subfebrile Temperaturen, Hämoptoë und eine deutliche Spitzentuberkulose sich entwickelten, die unter Progression der Erscheinungen zu einer subakuten Lungentuberkulose und zum Tode führten. Die Magen. erscheinungen waren in den letzten Wochen vollständig zurückgetreten.

Die Obduktion ergab tuberkulöse Bronchopneumonie beider Lungen mit chronischer fibröser Spitzentuberkulose. Die Schleim -

1) M. Kl. 1916 Nr. 28. 
haut des Magens und Duodenums erwies sich als voll kommen in tak t. Nirgend.s Spuren von Erosionen oder Narben. Ich veranlaßte die Untersuchung des Vagus. Der rechte Nerv fand sich in dichte tuberkulöse Bronchialdrüsen eingebettet und. komprimiert. Die mikroskopische Untersuchung beider Vagi (Prof. Marburg, Neurol. Univers.-Inst.) zeigte am rechten Vagus s tarke A tro phie mit starker Verdickung des Bindegewebes. Auch links Erscheinungen beginnender A trophie und Bindegewebsanreicherung. Narben.

Zwei andere Fälle meiner Beobachtung möchte ich auch hier anführen, weil ich überzeugt bin, daß gleichsinnige Wahrnehmungen zu einer wertvollen Korrektur unserer Anschauungen in der klinischen Beurteilung vieler Erkrankungsfälle beitragen können.

Fall 2. Frau H. v. R., 42 Jahre, Private, wurde mit einer schweren Meläna, hochgradiger Anämie (Hämoglobingehalt 40) im Juli 1913 an meine Abteilung gebracht. Seit dem Jahre 1892 wiederholt drückende Schmerzen in der Magengegend bei Tag und. bei Nacht mit Ausstrahlungen in den Rücken. Im Jahre 1907 stärkeıe Schmerzen, angeblich unabhängig von den Mahlzeiten, die aber durch Nahrungsaufnahme gebessert werden. Appetit immer gut, kein Erbrechen, kein Brechreiz. Im Mai 1913 unvermittelt, nachdem sie sich ein Jahr wohl gefühlt hatte, Bluterbrechen, später auch blutige und schwarze Stühle. Im Laufe der letzten fünf Jahre Gewichtsabnahme um $18 \mathrm{~kg}$. Die hochgradig anämische Patientin hatte anfangs noch längere Zeit bluthaltige Stühle. Es bestanden Druckschmerzen, namentlich im rechten Oberbauch. Unter der Annahme einer schweren Darmblutung, wahrscheinlich von einem Duodenalgeschwür ausgehend, wurde konservative Behandlung eingeleitet, die zu einer vollkommenen Besserung und Entlassung Ende September führte. Bei der zweiten Aufnahme, im Oktober 1913, charakterisiert sie ihre Schmerzen bereits nach dem Typus der Moynihanschen Beschwerden. Die damalige Mageninhalts prüfung ergab normalen Befund, Fehlen von Blut. Eine von mir vorgenommene Röntgenúntersuchung zeigte einen hypertonischen Hakenmagen mit deutlicher Druckempfindlichkeit am Pylorus, normal konfiguriurtem. Bulbus duodeni. Okkultes Blut negativ. Eine Kontrolluntersuchung (Prof. Holzknecht) am 31. Oktober ergab: Magenbefund ohne Anhaltspunkte für grobe Wandveränderungen, dagegen der Tonus erhöht, Anfangsentleerung gesteigert. Das Duodenum ist einer gröberen Veränderung sehr verdächtig an der Pars superior. Diese stimmt mit den von Cole beschriebenen Defekten bei kallösem Ulkus gut überein. Druckempfindlichkeit am Duodenum (tief) vorhanden, doch fällt sie nicht genau mit den anscheinenden Defekten zusammen. Im November 1913 neuerliche Aufnahme der Patientin. Wegen andauernder Schwäche und anhaltender Magenbeschwerden, des hochgradigen Verdachtes auf chronisches Duodenalgeschwür, wird die Patientin behufs Operation auf die Abteilung Prof. Clairmont verlegt.

Bei der Opera tion zeigen sich oberflächliche Adhäsionen am pylorischen Teil und der Pars descendens duodeni. Die Stelle eines Ulkus am Duodenum von außen nicht wahrzunehmen. Resektion der Pars pylorica des Magens und der Pars superior duodeni, $8 \mathrm{~cm}$, Naht. Gastroenterostumia r. p. Die Besichtigung des resezierten Magen-Duodenalanteiles zeigt keinerlei makroskopische Veränderungen. Glatter Heilungsverlauf. Entlassung am 26. November 1913.

Seitdem steht die Patientin andauernd in meiner Beobachtung. Es entwickelte sich unter Fieber und Abmagerung eine chronische Tuberkulose im rechten oberen und Mittellappen, die schwieligen Charakter zeigt und nach wiederholten Freiluft- und Ernährungskuren zur Erhaltung eines andauernd leidlichen Zustandes der Patientin gebracht werden kann. Vor kurzem hat sich die Kranke nach Davos begeben.

Fall 3. Ein dritter, durch die Operation verifizierter Fall betrifft einen Soldaten J. T. Aufnahme am 26. Oktober 1916. Im 18. Lebensjahre Lungenspitzenkatarrh. Im Jahre 1915 während drei Monaten im Spital mit Durchfall und Bauchschmerzen. Vor einem Monat heftige Schmerzen in der Magengegend und in der linken Bauchseite. Die Untersuchung ergibt eine Affektion der rechtseitigen Lungenspitze. Okkulte Blutung zweimal negativ, einmal positiv. Mageninhalts. prüf u ng ergibt Spuren von Blutfarbstoff. Untersuchung auf Retention (Faber-Bourget) negativ. Röntgenbefund: Hypertonischer, hochstehender Magen, große Kurvatur $2^{1 / 2}$ Querfinger über dem Nabel. Bulbus scharf umschrieben, normal konturiert, deutliche Druckempfindlichkeit in der Regio peripylorica, auch auf den Bulbus duodeni übergreifend. Dieser, sowie der Magen, nach links nicht dislozierbar. Diagnose: Adhaesiones peripyloricae ex ulcere. Wegen andauernder, kontinuierlicher Schmerzen wird Patient zur Probelaparotomie überwiesen. Am 9. Februar (Prof. Glairmont) an Magen, Duodenum und Gallenblase vollständig normaler Befund. Keine Ad.häsionen.
Gegen die Appendix zu scheinbare Verwachsungen. Da sie nicht leicht zu erreichen ist, wird von der Entfernung abgesehen.

Seitdem hat sich, ohne daß es zur Operation kam, die Zahl ähnlicher Beobachtungen sehr vermehrt. Doch haben wir aus den Erfahrungen gelernt und wissen heute, daß solche Fälle, welche die Geschwürssymptome so auffallend nachahmen, von einer anatomischen Erkrankung des Vagus oder von zen. tralen Residuen einer Hilusaffektion ausgehen, resp. von einer vagotonischen Disposition herrühren können.

In einer weiteren Mitteilung ${ }^{1}$ ) habe ich gezeigt, daß Kardialgie, Erbrechen und Dyspepsie, auch Bluterbrechen im Vordergrunde, eine latente oder aktuelle Tuberkulose der Lunge im Hintergrund, für dieses klinische Syndrom charakteristisch sind.

Ich habe auf das familiäre Vorkommen solcher gastrischer Formen der Tuberkulose oder der tuberkulösen Disposition hingewiesen und gezeigt, daß Potain in einer Arbeit Mortows aus dem Jahre 1737 die initiale Dyspepsie der Tuberkulose mit einer Irritation des Pneumogastrikus erklärt. Kompression des Vagus durch tuberkulöse Drüsen haben Potajn und Guéneau de Muss y schon im Jahre 1861 nachgewiesen. Dieses Syndrom habe ich mit der Pathogenese des Magengeschwürs und den bekannten experimentellen Untersuchungen von Schiff, Ebstein, Brown-Séquard, Talma, Lichtenbelt, Bergmann, Westphal und Katsch in Verbindung gebracht.

Aus der anfänglichen funktionellen Erkrankung, Hypertonie, Kardio-Pylorospasmus, kann am Ende der Entwicklungsreihe das Magen- und Duodenalgeschwür hervorgehen. Dies nur nebenbei. Wichtig ist die Kenntnis dieser Krankheitsformen, weil die so schwierige Differentialdiagnose zwischen funktionellen Magenerkrankungen und dem Magen- und Duodenalgeschwür von diesen Beobachtungen Notiz nehmen muß. Klinisch und radiologisch ausgeprägte Ulkussymptome am Magen (auch Blutungen) können bei vollkommen intaktem Magen-Duodenaltrakt durch eine Art von Spasmophilie, durch Reizung des Vagussystems, hervorgerufen sein und die klinische Spitzmarke einer latenten oder larvierten Lungentuberkulose darstellen. Die Häufigkeit dieses Syndroms ist durch nachträg. liche Untersuchungen von Reitter ${ }^{2}$ ) bestätigt worden.

Aus dieser innigen, auch durch detaillierte Untersuchungen oft schwer zu differenzierenden Beziehung zwischen Funktionsstörung einerseits, anatomischer Erkrankung (Ulkus) anderseits ist zu ersehen, mit welchen Schwierigkeiten die Röntgen. diagnose des Duodenalgeschwürs zu rechnen hat. Im Jahre 1913 hat Ha u de $\mathrm{k}$ auf dem Chirurgenkongreß folgende Röntgensymptome des Ulcus duodeni aufgezählt: 1. konstanten Schatten am oberen Duodenum, 2 . die Nische, 3 . den persistierenden Duodenalfleck (Dauerbulbus, der Duodenalzapfen von Bier), 4. den umschriebenen, dem Bulbusschatten entsprechenden Druckpunkt, 5. die duodenale Magenmotilität (Kreuzfuchs). Sie besteht in einer vermehrten Anfangsentleerung mit folgender Retention (Haudek).

Auch diese Motilitätsanomalie hat schon Kreuzfuchs selbst als nicht pathognomonisch für das Duodenalgeschwür angesehen, sondern auch bei sekundären Duodenalstörungen, ausgehend von Erkrankungen der Gallenblase, des Pankreas, des Wurmfortsatzes, gefunden.

Bei einer kritischen Sichtung dieser Thesen zeigt sich, daß sie für die Erkenntnis des Duodenalgeschwürs nicht ausreichen, daß sie vielmehr vieldeutig sind und, wie v. Bergmann schon gesagt hat, ,nicht das Ulkus selbst, sondern geänderte Funktionen dieseits und jenseits des Pylorus aufdecken". Die zwei von v. Bergmann und seinen Schülern Westphal und Katsch aufgestellten Typen, der hyperperistaltische und der maximalsekretorische Typus, sind nur funktionelle Zeichen, welche bei reinen Neurosen und, wie die Operationserfahrungen Westphals zeigen, bei der Reichmannschen Krankheit ohne Ulkus vorkommen. Der Satz Moynihans, daß Hypersekretion Ulkus bedeute, kann nach den neueren, durch die Operation sichergestellten Beobachtungen nicht aufrechterhalten werden. Und so sehen wir, da B eigentlich erst die Untersuchungen von David (Halle) und Holzknecht (unmittelbare Auffüllung und Darstellung des Duodenums mittels der Duodenalsonde und mittels Kompressionsblende), ferner die Untersuchungen der Amerikaner Cole, George und Gerber und

i) W. kl. W. 1917 Nr. 20. — 2) W. kl. W. 1917 Nr. 20. 
Freud, welche durch Serienaufnahmen Wanddefekte, Narben, Divertikelbildung am stenosierten Bulbus duodeni aufdeckten, die durch das Ulikus gesetzten anatomischen Veränderungen zur direkten Anschauung bringen. Eine verbesserte Methodik der Untersuchung verdanken wir Chaoul und Stierlin, ${ }^{1}$ ) welche als charakteristisehe Formanomalien anführen: Den Bulbusdefekt, den Pylorusfortsatz, Pylorussporn, Sanduhr-Bulbus, persistierenden Bulbus, persistierenden Ampullenfleck und parabulbäre Flecke. Die Aufnahmen erfolgen in verschiedenen Körperlagen und in wiederholten kurzfristigen Intervallen.

Nach den zahlreichen Erfahrungen unter Kontrolle der Biopsie kann ich mich unbedingt dem Standpunkte Stierlins anschließen, der die erstgenannten Symptome als indire kte und funktionelle, in ihrer Valenz hinter die direkten, durch die unmittelbareDarstellung der anatomischen Wandveränderung nachgewiesenen Symptome zurückstellt. Wenn überhauptdie Rö̈ntgenuntersuchung des Duodenalgeschwürs in der Diagnostik dieser Erkrankung einen ersten Platz einnehmen oder behaupten soll, muß sie auf den Ausbau der direkten Methoden ihr Schwergewicht legen.

Aus einer Sammlung meiner Fälle von Duodenalulkus möchte ich die durch Operation oder Autopsie sichergestellten in einer Gruppe zusammenfassen. Sie betrifft 33 Fälle, von denen 15 von vornherein auszuscheiden sind, weil sie, mit schwerer Blutung oder Perforation eingeliefert, von der Röntgenuntersuchung ausgeschlossen waren. Bei 15 Fällen wurde die klinische Diagnose auf Duodenalgeschwür durch die Operation bestätigt. Davon war in 4 Fällen der Röntgenbefund typisch, mit Coleschem Defekt; 8 mal war der Röntgenbefund unvollständig, 2 mal negativ, 1 Fall wurde nicht untersucht, 3 mal ergaben sich Fehldiagnosen bei radiologisch-positivem Befunde (Cole und Einziehung am Pylorus).

Wenn ich auch nicht alle diese Befunde einheitlich verwerten will, weil sie aus verschiedenen Entwicklungsphasen des Röntgenverfahrens stammen, so lehren sie doch, ebenso wie die große Zahl der klinisch einwandfreien, nicht perlustrierten Fälle, die ich beobachtet habe, daß die bisherigen Röntgenzeichen für das Duodenalgeschwür allein nicht'ausreichen, weil sie entweder bei vorhandenem Geschwür versagen oder oft zur Annahme eines Geschwürs verleiten, wo nur funktionelle Veränderungen vorliegen. Es bleibt noch abzuwarten, ob auch die Bilder nach dem Verfahren von Chaoul und Stierlin immer der Ausdruck anatomischer Wandveränderungen sind, oder ob sie nicht in einer gewissen Anzahl von Fällen nichts anderes zeigen als Phasen in der Peristaltik des Duodenums.

Diese Verhältnisse haben bei einem Teile der Radiologen zu einer Reaktion geführt. Das kallöse Magengeschwür mit seinem Nischensymptom, der narbige Sanduhrmagen sind prima vista-Leistungen der Röntgentechnik, die ja auch auf anderen Gebieten über gewisse Schnellmethoden der Diagnostik verfügt. $\mathrm{E}_{\mathrm{S}}$ wäre $\mathrm{zu}$ schön gewesen, wenn solche unmittelbare Erfolge auch beim schwierigen Kapitel des Duodenalgeschwürs zu erzielen gewesen wären. Das kam anders Und so sehen wir die Radiologie bei einer gewissen Atempause in ihrer Entwicklung angelangt. Was wir bei einer exakten Methode gern vermissen, das Hineindeuten und Kommentieren wird jetzt bei der Radiologie des Verdauungstraktes zu einer schlechten Gewohnheit. Immer mehr hört man Hinweise auf die funktionelle Komponente im Krankheitsbilde des Ulkus von Radiologen vorgebracht, für die die spasmogene Theorie v. Bergmanns zu einem Rettungsanker geworden ist. Für alle Freunde der Radiologie bedeutet diese Haltung eine gewisse Enttäuschung, weil sie diesem bedeutungsvollen Hilfsorgan der Klinik eine andere Rolle zugeschrieben haben.

Wenn ich mir die Entwicklungsstufen der Magendarmpathologie in den letzten Jahrzehnten vor Augen führe, so können wir folgende Stadien darin unterscheiden: Anfangs die unentwegte Vorherrschaft anatomischer Vorstellungen, mit der Einführung der Mageninhaltsuntersuchung lernt man die Neu. rosen und funktionellen Erkrankungen kennen, schätzen und überschätzen. Das ging so weit, daß mit der Ueberwertung der funktionellen Ergebnisse eine Verfälschung der Auffassungen Platz gegriffen hat, sodaß man überall nur mehr Neurosen sah

1) M. m. W. 1917 Nr. 48. und die altbewährten, anatomisch fundierten Krankheitsbilder verdrängt wurden. Gerade die Lehre vom Ulkus ist dabei am schlechtesten weggekommen. Vor lauter Hyperazidität, Hypersekretion, Gastrosukkorrhoe und alimentärer, digestiver Sekrètion übersah man die alt fundierte Tatsache, daß das Ulkus eine häufige Krankheit ist. Erst die zunehmende Zahl der Operationen hat hier eine Wandlung der Anschauungen angebahnt. Von der Radiologie, der Biopsie bei geschlossener Bauchhöhle, haben wir eine durchgreifende Korrektur dieser in eine falsche Richtung geratenen klinischen Anschauungen erwartet. Das Röntgenverfahren kann aber nur dann einen fördersamen Einfluß auf die Entwicklung der Klinik gewinnen, wenn die Radiologen nicht bloß Techniker sind, sondern ihre Befunde pragmatisch verarbeiten und der Klinik nutzbar machen, d. h. mit den Augen des Klinikers sehen und mit der durch die Erfahrungen der Klinik vorgezeichneten Kritik verwerten. Ein selbstherrliches Hinauswachsen über das ihr zukommende Betätigungsgebiet kann der Radiologie selbst mehr als der Klinik zum Schaden gerejchen. Wo die Grenze für die Erkennungsmöglichkeiten der Radiologie derzeit noch besteht, muß sie die Entscheidung an die Klinik verweisen, $d . h$. an die Stelle, wo die innige und allseitige Beobachtung des Kranken der oberste Grundsatz ist. Denn die Klinik zieht das Resümee aus allen Befunden und Untersuchungsmethoden, sie entscheidet nicht bloß nach festen Ergebnissen, ihre Urteile sind auch getragen von der Summe an Tradition und Einzelerfahrung, welche die ärztliche Tätigkeit über das Niveau der automatischen Arbeit hinaus zur ärztlichen Kunst erhebt. 